$1989 \mathrm{dec} \bullet$ volume $63 \bullet$ no. $4 \bullet$ pages $269-360$ ISSN 0022 149x

\title{
Journal of Ielminthology
}

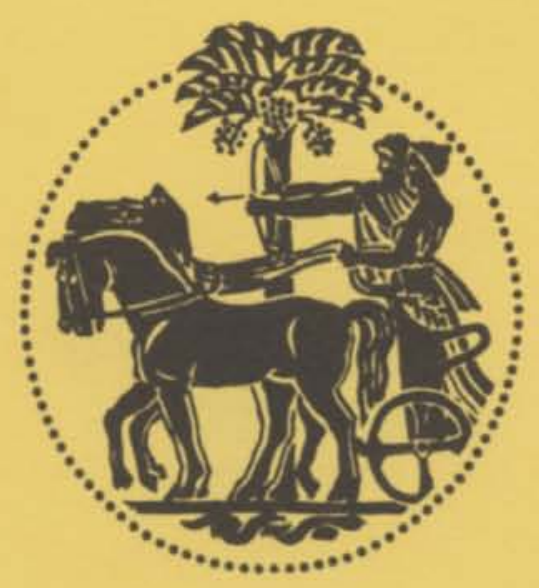




\section{JOURNAL OF HELMINTHOLOGY}

(Founded by R. T. Leiper in 1923 and edited from 1946-72 by J. J. C. Buckley, from 1972-80 by $R$. Muller and from 1980-86 by S. Willmott)

EDITOR: Dr. Ralph Muller and Dr. Lotfi F. Khalil

Editorial Assistant: Hilary White

CAB International Institute of Parasitology,

395A Hatfield Road,

St Albans, Herts AL4 0XQ, England.

Subscription enquiries should be sent to:

Bureau of Hygiene \& Tropical Diseases,

Keppel Street,

London WC1E 7HT, England.

Advertisement enquiries should be sent to:

Ms Dora Curtis,

Professional Journals Advertisement Representations Ltd,

Sudley Chambers,

8 Sudley Road,

Bognor Regis,

Sussex PO21 1EU, England.

Published in 4 parts per year.

Back volumes should be ordered from Wm. Dawson \& Sons Ltd., (Back Issues Dept.), Cannon House, Folkestone, Kent CT195EE, England. Priceson application. 